\title{
Criblage de molécules chimiques actives contre Pseudomonas syringae pv syringae sur plants de vigne cultivés in vitro et in situ
}

\author{
JL Gaignard, J Luisetti
}

\begin{abstract}
INRA, centre d'Angers, station de pathologie végétale et phytobactériologie, 42 rue G Morel,
\end{abstract} BP 57, 49071 Beaucouzé Cedex, France

(Reçu le 2 décembre 1991; accepté le 14 avril 1992)

\begin{abstract}
Résumé - Une méthodologie, basée sur l'utilisation de vitroplant de vigne, destinée à estimer la capacité de molécules chimiques à réduire les populations épiphytes de $P s$ pv syringae, a été mise au point; les résultats des essais conduits soit sur des vitroplants axéniques, soit sur des vitroplants transférés en acclimatation et placés en conditions aseptiques ou non, et destinés à comparer l'efficacité de l'oxytétracycline, de la kasugamycine, de la fluméquine, de la bouillie bordelaise et du mancozèbe, concordent avec ceux obtenus sur des plants de vigne conduits en conteneurs. Dans ces essais, la kasugamycine a montré une bonne efficacité vis-à-vis de $P s$ pv syringae; la fluméquine une efficacité moyenne; la bouillie bordelaise une efficacité limitée et variable selon les essais et le mancozèbe aucune efficacité. La technique de criblage de l'efficacité antibactérienne de molécules chimiques peut être développée sur plants en acclimatation en microserre, conduits en conditions subaseptiques; elle se situe entre le test réalisé en boîte de Petri (détermination de la concentration minimale inhibitrice) et l'essai de plein champ.
\end{abstract}

\section{Pseudomonas syringae pv syringae / culture in vitro / lutte chimique / méthodologie / vigne}

Summary - A method using in vitro and in situ grapevine plants to screen chemicals active against Pseudomonas syringae pv syringae. Pseudomonas syringae pv syringae occurs as an epiphyte on the surface of grapevine, particularly on buds during spring. As it is also an ice nucleation active bacterium, it is considered as one of the factors causing spring frost damage to the grapevine. Some protection against frost injury could be achieved by reducing the level of $\mathrm{P} s \mathrm{pv}$ syringae epiphytic populations. The chemicals available for control are limited to some copper formulations and antibiotics, both of which have some disadvantages. More efficient bactericides are required and consequently a great number of chemicals have to be tested. A method using grapevine in vitro plants has been developed to estimate the activity of molecules in reducing the epiphytic populations of $\mathrm{P} s \mathrm{pv}$ syringae; the results of the experiments performed either on in vitro plants (fig 1) or on plants transferred to acclimatation (figs 2 and 3), and aimed at comparing the efficacy of oxytetracyclin, kasugamycin, flumequine, Bordeaux mixture and mancozebe agree with those obtained on plants grown in containers (fig 4). This method could first be extended to all the strains of the syringae pathovar whatever the host plant and then to the screening of chemicals active against any phytopathogenic bacterium of the genus Pseudomonas or Xanthomonas in so far as it has a significant epiphytic phase within its biological cycle.

\section{Pseudomonas syringae pv syringae / in vitro culture / chemical control / methodology/grapevine}

\section{INTRODUCTION}

Pseudomonas syringae pv syringae, bactérie épiphyte, notamment sur vigne et sur arbres fruitiers, est présente en quantité importante à la surface des bourgeons et des jeunes feuilles en développement au printemps (Crosse, 1959; English et Davis, 1960; Dowler et Petersen, 1967; Ercolani 1969; Luisetti et Paulin, 1972; Gardan et al, 1973; Young, 1978; Luisetti et
Gaignard, 1987a, b; Malvick et Moore, 1988; Mansvelt et Hattingh, 1988; Hattingh et al, 1989). De plus $P S$ pv syringae est une bactérie glaciogène douée de la capacité d'induire la rupture de surfusion de l'eau à des températurs négatives proches de $0{ }^{\circ} \mathrm{C}$ (Maki et al, 1974; Arny et al, 1976; Vali et al, 1976; Hirano et al, 1978; Lindow et al, 1978a, b; Maki et Willoughby, 1978; Paulin et Luisetti, 1978; Yankofsky et al, 1981; Gross et al, 1983; Luisetti et Gaignard, 
1987a). Lors d'expérimentations en conditions contrôlées, Luisetti et al (1991), ont démontré sur vigne que cette bactérie avait un rôle dans l'initialisation de la cristallisation de l'eau des bourgeons. La réduction du nombre de ces germes glaciogènes à la surface des jeunes organes de vigne devrait donc permettre de diminuer les risques de gel sur ces organes herbacés particulièrement fragiles.

Parmi les pesticides actuellement homologués, on ne dispose pas de bactéricides efficaces; les sels de cuivre n'ont qu'un effet limité et présentent à la fois des risques de phytotoxicité (Prunier et al, 1974; Lindow, 1983; Lindow et Connel, 1984; Luisetti et al, 1984) et des risques de sélection de souches résistantes aux métaux lourds (Sundin et al, 1989; Lindow, 1991); les antibiotiques ne sont utilisables que dans des cas particuliers (Brisset et al, 1991); ils offrent aussi des risques de sélection de bactéries résistantes (Chabbert, 1973; Manceau, 1984; Goto, 1991; Sobiczewski et al, 1991). Leur emploi ne sera probablement jamais autorisé en France dans le contexte de la lutte antigel (Coléno et al, 1982). La lutte chimique ne peut donc aujourd'hui permettre d'abaisser les populations bactériennes glaciogènes au printemps. Les perspectives de lutte biologique (compétition microbiologique à la surface des bourgeons et des feuilles) paraissent encore éloignées et aléatoires (Andrews, 1985; Leben, 1985; Luisetti et Gaignard, 1986; Lindow, 1988).

La recherche de nouvelles molécules à effet bactéricide totalement inoffensives pour l'homme et l'environnement demeure donc un objectif immédiat et ne concerne pas seulement un seul pathovar de syringae mais toutes les bactéries phytopathogènes, épiphytes ou non, glaciogènes ou non (Coléno et al, 1982). Ceci nécessite le criblage d'un grand nombre de molécules supposées avoir un pouvoir antibactérien. Le test in vitro, c'est-à-dire la confrontation directe de la molécule et du microorganisme ne suffit pas pour juger l'efficacité antibactérienne d'une molécule dans le contexte de la culture. La réalisation d'essais sur plantes entières est donc indispensable; l'efficacité peut être estimée d'abord dans le cadre d'essais parcellaires puis éventuellement confirmée au travers d'essais de plein champ selon la méthodologie proposée par Gaignard et al (1973). Ce schéma d'étude exige de l'espace et du matériel végétal.

L'utilisation de vitroplants paraît particulièrement adaptée à ce type d'étude et en particulier au criblage de molécules chimiques. Le vitro- plant de poirier a déjà été utilisé pour rechercher des molécules actives contre $E$ amylovora, agent responsable du feu bactérien (Brisset, 1990). PS pv syringae, est un autre modèle pour lequel le vitroplant pourrait être utilisé. Nous avons entrepris le criblage des molécules actives contre $P s$ pv syringae, au travers d'une analyse de leur effet sur la dynamique épiphyte de cette bactérie installée sur des vitroplants de vigne soit maintenus en bocaux, soit placés en acclimatation, en conditions subaseptiques ou en atmosphère normale. Un essai sur plants en pots, mené en parallèle, a servi de référence pour comparer les résultats obtenus avec ce matériel miniaturisé.

\section{MATÉRIEL ET MÉTHODES}

\section{Matériel végétal}

Des vitroplants du clone $41 \mathrm{~B}$, hybride Vitis vinifera $\mathrm{x}$ Vitis berlandieri, sont utilisés pour l'essai réalisé en bocaux et des vitroplants du cépage Merlot appartenant à l'espèce Vitis vinifera pour les 2 essais réalisés en acclimatation. Les vitroplants sont cultivés sur le milieu de Murashige et Skoog (1962) modifié en ce qui concerne les macroéléments : (mg |-1) $\mathrm{NH}_{4} \mathrm{NO}_{3}$ $(412,5), \mathrm{KNO}_{3}$ (950), $\mathrm{CaCl}_{2}$ (165), $\mathrm{MgSO}_{4}, 7 \mathrm{H}_{2} \mathrm{O}(185)$, $\mathrm{KH}_{2} \mathrm{PO}_{4}(85)$, soit gélosé, soit liquide mais alors avec un support synthétique de type Milcap ${ }^{\circledR}$ (ChalletHérault, Nuaillé 49). Le pH est ajusté à 5,5-6 avant autoclavage. L'essai réalisé sur plants en pots est conduit sur le cépage Chardonnay appartenant à l'espèce Vitis vinifera. II s'agit de plants greffés sur le clone 41B. Le matériel végétal a été choisi en fonction des disponibilités de nos fournisseurs.

L'essai 1 est réalisé sur vitroplants au stade 6-8 feuilles, conduits en bocaux et installés dans une cellule de vitroculture à $24 / 19^{\circ} \mathrm{C}$, avec une photopériode de 16/8 h. Cinq bocaux contenant 8-10 plants sont réservés à chaque traitement.

L'essai 2 est mis en place sur des vitroplants en phase d'acclimatation mais maintenus sous conditions subaseptiques : les vitroplants du cépage Merlot

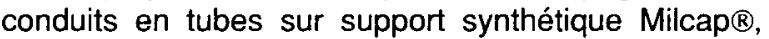
sont inoculés au stade 6-8 feuilles. Huit jours après, ils sont sortis du tube en conditions d'asepsie (sous hotte à flux laminaire) pour être installés sur une plaque pourvue d'alvéoles dans lesquelles sont introduits les supports synthétiques. Les supports et les racines ne sont pas rincés afin de conserver le milieu de culture. Quelques $\mathrm{ml}$ de ce milieu sont d'ailleurs ajoutés sur le support au moment du repiquage pour favoriser la croissance des plants. La plaque alvéolée contenant les plants est alors installée dans une microserre de marque Plantpak (Noyon 60), d'un volume de 2 I, et à l'intérieur de laquelle sont maintenues des conditions subaseptiques. Chaque microserre comprenant 24 plants, est réservée pour chaque traitement. Les mi- 
croserres et les plaques alvéolées sont préalablement stérilisées à l'alcool et par exposition durant une nuit aux rayons ultraviolets $(254 \mathrm{~nm})$. Durant le transfert, les plants sont en permanence brumisés avec de l'eau stérile. Les brumisations sont fréquentes les premiers jours pour éviter le dessèchement des plants, puis le couvercle est progressivement levé. Les microserres sont installées dans la cellule de vitroculture, donc en atmosphère microbiologiquement peu polluée. Après l'apport des produits de traitements, les plants sont brumisés une fois par jour.

L'essai 3 s'est déroulé pendant la phase d'acclimatation. Les vitroplants conduits en tubes sur support

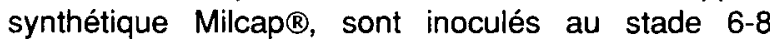
feuilles. Huit jours après, ils sont sortis du tube (rupture de l'asepsie), le support et leurs racines sont rincés à l'eau, et ils sont alors installés dans une miniserre de marque BHR (St-Germain-du-Plain 71), d'un volume de $10 \mathrm{I}$, sur un mélange terreux détrempé. II s'agit d'un mélange composé de sable de Loire (1/3), de terreau NF-U-44551 (1/3) et de tourbe NF-U-44051 $(1 / 3)$. Au cours du transfert, une brumisation des plants est pratiquée comme dans l'essai 2, mais dans ce cas, on utilise l'eau du réseau urbain. Une miniserre comprenant 30 plants est préparée pour chaque traitement. Les miniserres sont installées dans une cellule climatisée à $20^{\circ} \mathrm{C}$ (précision $\pm 1^{\circ} \mathrm{C}$ ) avec une photopériode de $16 / 8 \mathrm{~h}$.

L'essai 4 est effectué sur des plants de vigne conduits en conteneurs de 4 I sur un mélange terreux identique à celui utilisé pour l'essai 3 . Après débourrement ils sont maintenus en serre jusqu'au stade 15-20 feuilles. Ils sont ensuite installés dans la même cellule climatisée que les plants de l'essai 3 . Un lot de 10 plants est réservé pour chaque traitement. La pulvérisation des bactéries est réalisée 4 j après la mise en place des plants en cellules climatisées.

L'intensité lumineuse, dans les cellules de culture, est de 3000 lux.

\section{Matériel microbiologique}

La souche bactérienne INRA JF $7684 \mathrm{Sm}^{r}$ a été utilisée dans les 4 expériences. C'est un mutant spontané, résistant à la streptomycine, sélectionné à partir de la souche de $P$ s pv syringae INRA JF 768 4, elle-même isolée d'un lavage de bourgeon de vigne provenant de Champagne. La souche est glaciogène et appartient à l'un des biotypes les plus fréquemment isolés sur vigne (Luisetti et Gaignard, 1987a). La résistance à l'antibiotique permet d'utiliser un milieu sélectif pour récupérer les bactéries des plantes et ainsi d'abaisser le seuil de détection.

\section{Méthodes et traitements}

La souche est multipliée sur le milieu LPGA : extrait de levure BioMérieux (7 g. $\left.\left.\right|^{-1}\right)$, Biogélytone BioMérieux (7 g. $\left.\mathrm{I}^{-1}\right)$, Glucose monohydrate Prolabo (7 g. $\left.\mathrm{I}^{-1}\right)$, Agar
Agar type A Labo Ind Biol (15 g..$\left.^{-1}\right)$ et $\mathrm{pH}$ ajusté à 7 avant autoclavage. Après $48 \mathrm{~h}$ d'incubation à $24^{\circ} \mathrm{C}$, l'inoculum est préparé par une mise en suspension des bactéries dans de l'eau distillée stérile et ajustée à la concentration de $10^{8}$ bactéries par ml. L'inoculation des plants correspond à l'installation de la bactérie à la surface des feuilles soit par dépôt, soit par pulvérisation. L'un est réalisé en ambiance stérile (hotte à flux laminaire) par dépôt, à l'aide d'une micropipette, de microgouttes de $20 \mu$ sur les feuilles des vitroplants. L'autre concerne exclusivement les plants en pots, et est réalisée dans la cellule climatisée à l'aide d'un pulvérisateur à jet porté de $5 \mathrm{I}$. L'inoculation dans l'un ou l'autre cas ne conduit jamais à l'expression de symptômes sur les plants de vigne.

Quelques jours après l'inoculation, des répliques de feuilles sont réalisées sur plaques de milieu $B$ deKing et al (1954). II s'agit d'un simple contact des faces supérieure et inférieure de feuilles avec le milieu permettant d'observer, après $48 \mathrm{~h}$ à $24^{\circ} \mathrm{C}$, la densité et la répartition des populations bactériennes. Lorsque la colonisation se révèle bonne, les produits à étudier sont alors appliqués. Cette application a eu lieu, pour les essais 1 et $4,8 j$ après l'apport des bactéries et, pour les essais 2 et $3,8 \mathrm{j}$ après la mise en acclimatation. Les produits, dilués dans de l'eau, sont appliqués à l'aide d'un fin vaporisateur pour l'essai 1 et d'un pulvérisateur de $500 \mathrm{ml}$ pour les essais 2, 3 et 4 .

Dans chacun des 4 essais, les 5 mêmes produits sont mis en comparaison; il s'agit de :

- 3 antibiotiques : l'oxytétracycline (tétracycline, Mycoshield $\circledast$, Pfizer, New York), la kasugamycine (aminoglycoside, Kasumin $\circledast$, Hokko Chemical Industry Co, Ltd, Tokyo), la fluméquine (quinolone, Firestop( 8 , 3M Santé, Pithiviers), aux doses respectives de 1000 , 1000 et 300 ppm de MA.

- 1 formulation à base de cuivre : la bouillie bordelaise (sulfate de cuivre, Raffineries de soufre réunies, Marseille) dosée à 1250 ppm de Cu métal,

- 1 fongicide de synthèse : le mancozèbe (dithiocarbamate, Dithane M45, La littorale, Béziers) à la dose de 1000 ppm de MA.

Des plants témoins inclus dans les essais reçoivent une pulvérisation d'eau, en guise de traitement.

\section{Analyse et traitement des résultats}

La dynamique des populations de $P$ s pv syringae à la surface des plants est déterminée au travers d'estimations réalisées pendant la durée des essais. Une première estimation est effectuée juste avant l'application des produits de traitement, les autres étant étalées dans le temps, jusqu'à $14 \mathrm{j}$ pour les essais 1 et $3,7 \mathrm{j}$ pour l'essai 2 et $9 \mathrm{j}$ pour l'essai 4 . Les estimations sont faites sur des échantillons de 10 feuilles pour les essais sur vitroplants en bocaux ou en acclimatation et de 30 feuilles pour l'essai sur plants en pots, la taille minimale de l'échantillon ayant été définie par une étude préalable. L'échantillon est broyé soit pen- 
dant $10 \mathrm{~s}$ et dans $10 \mathrm{ml}$ d'eau distillée stérile à l'aide d'un Ultra Turax $\AA$, soit pendant $1 \mathrm{~min}$ et dans $300 \mathrm{ml}$ d'eau distillée stérile à l'aide d'un Waring Blendor ${ }^{\circledR}$. Une partie aliquote $(50 \mu l)$ du broyat et de chacune de ses premières dilutions au $1 / 10$ est étalée sur une plaque de milieu B de King (1954) additionnée d'un fongicide (cycloheximide, Sigma) et de streptomycine (Sigma) à la dose de $50 \mathrm{mg} \cdot \mathrm{ml}^{-1}$. Les colonies sont dénombrées après $48 \mathrm{~h}$ d'incubation à $24^{\circ} \mathrm{C}$. La population est exprimée en nombre de bactéries par organe dans le texte et sous forme de son logarithme dans les graphiques.

Pour rendre plus aisée la comparaison des dynamiques des populations à la surface des plants traités et des plants témoins, les populations initiales estimées sur les échantillons prélevés avant l'apport des produits sont, pour chaque lot, ramenées à un même niveau qui est la moyenne des populations de l'ensemble, puis les niveaux de population pour chaque produit et pour chaque contrôle sont alors recalculés.

Une statistique d'ordre (test de Kramer, Kahan et al, 1973) est appliquée sur le classement des produits, permettant de les situer éventuellement dans des groupes d'efficacité différente (tableau I).

\section{RÉSULTATS}

La réussite de l'installation de $P s$ pv syringae à la surface des vitroplants ou des plants en pots se manifeste par la présence, avant l'application des produits, de populations de l'ordre de $5 \times$ $10^{3}$ bactéries par feuille (essais 1 et 4 ) ou de $4 x$ $10^{4}$ bactéries par feuille (essai 3 ) ou de $3 \times 10^{5}$ (essais 2).
L'observation de l'évolution de la population sur les plants témoins pulvérisés avec de l'eau permet d'apprécier l'influence des conditions de l'essai sur la dynamique de $P s$ pv syringae. Dans l'essai 1 (en bocaux), la population s'est maintenue sur le témoin à un niveau stable durant les $14 \mathrm{j}$ ( $5 \times 10^{3}$ bactéries par feuille). Dans l'essai 2 (en microserres subaseptiques), les bactéries se sont maintenues à un niveau élevé sur les plants ( 3 à $5 \times 10^{5}$ bactéries par feuille). L'absence d'autres microorganismes et le rapport élevé surface foliaire/volume de la microserre entraînant une forte humidité, sont des éléments favorables à l'installation et la multiplication de $P s$ pv syringae. Une baisse importante des populations a été observée dans les 2 premiers jours de l'essai 3 conduit en miniserres (seulement $10^{2}$ bactéries par feuille); elle pourrait être reliée à la réduction du nombre de brumisations; les populations remontent ensuite au niveau de $5 \times 10^{3}$ bactéries par feuille. Sur les plants en pots, le niveau des populations s'est élevé durant l'essai pour atteindre $10^{6}$ bactéries par feuille sur les plants témoin dès le sixième jour.

\section{Essai conduit sur vitroplants en bocaux (essai 1; fig 1)}

La kasugamycine et la fluméquine manifestent rapidement leur efficacité antibactérienne qui se traduit par une non-détection de $P$ s pv syringae

Tableau I. Bilan des classements des produits selon le test de Kramer (seuil 5\%).

\begin{tabular}{|c|c|c|c|}
\hline Essai & Groupe 1 & Groupe 2 & Groupe 3 \\
\hline Essai 1 & Témoin eau & $\begin{array}{l}\text { Mancozèbe } \\
\text { Bouillie bordelaise } \\
\text { Oxytétracycline }\end{array}$ & $\begin{array}{l}\text { Fluméquine } \\
\text { Kasugamycine }\end{array}$ \\
\hline Essai 2 & $\begin{array}{l}\text { Bouillie bordelaise } \\
\text { Témoin eau }\end{array}$ & $\begin{array}{l}\text { Mancozèbe } \\
\text { Fluméquine } \\
\text { Oxytétracycline }\end{array}$ & Kasugamycine \\
\hline Essai 3 & Témoin eau & $\begin{array}{l}\text { Oxytétracycline } \\
\text { Mancozèbe } \\
\text { Bouillie bordelaise }\end{array}$ & $\begin{array}{l}\text { Fluméquine } \\
\text { Kasugamycine }\end{array}$ \\
\hline Essai 4 & Témoin eau & $\begin{array}{l}\text { Mancozèbe } \\
\text { Bouillie bordelaise } \\
\text { Fluméquine } \\
\text { Oxytétracycline }\end{array}$ & Kasugamycine \\
\hline
\end{tabular}


Log du nombre de P. s. s. par feuille

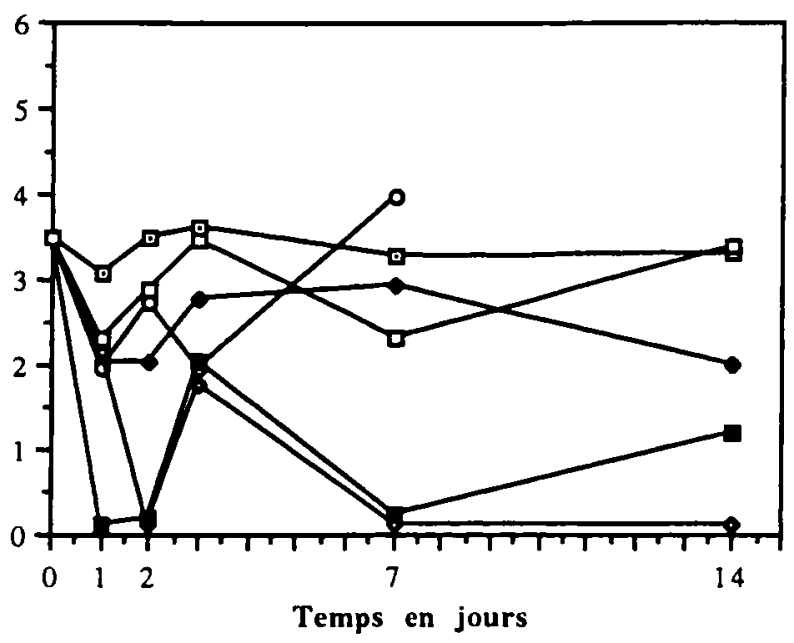

Fig 1. Évolution dans le temps de la population de $P s p v s y-$ ringae sur vitroplants conduits en bocaux (essai 1); en fonction de différents traitements. - $\odot-$ témoin eau; - - oxytétra; - O- cuivre; - 0 - kasugamycine; - - fluméquine; - [- mancozèbe.

dans les 2 j suivant l'apport des produits; malgré une légère remontée au $3^{\theta} \mathrm{j}$, les populations se maintiennent par la suite à un niveau très bas. La bouillie bordelaise réduit la population d'une puissance au cours des 3 premiers jours mais cet effet reste limité dans le temps puisque la population est, au $7^{e} j$, à un niveau supérieur. Une chute de feuilles, due à la phytotoxicité du cuivre, est constatée dans cet essai, ce qui n'a pas permis de poursuivre les analyses bactériennes. L'oxytétracycline et le mancozèbe ne réduisent que très modestement le niveau des populations qui se situent cependant au-dessous de celui du témoin.

L'application du test de Kramer sépare très nettement la kasugamycine et la fluméquine du témoin; le mancozèbe, la bouillie bordelaise et l'oxytétracycline sont dans un groupe intermédiaire.

\section{Essai conduit durant l'acclimatation, mais en conditions subaseptiques (essai 2; fig 2)}

La disparition des populations de $P s$ pv syringae consécutive à l'application de la kasugamycine est rapide et durable. L'oxytétracycline a entraîné également un abaissement du niveau bactérien
Log du nombre de P. s. s. par feuille

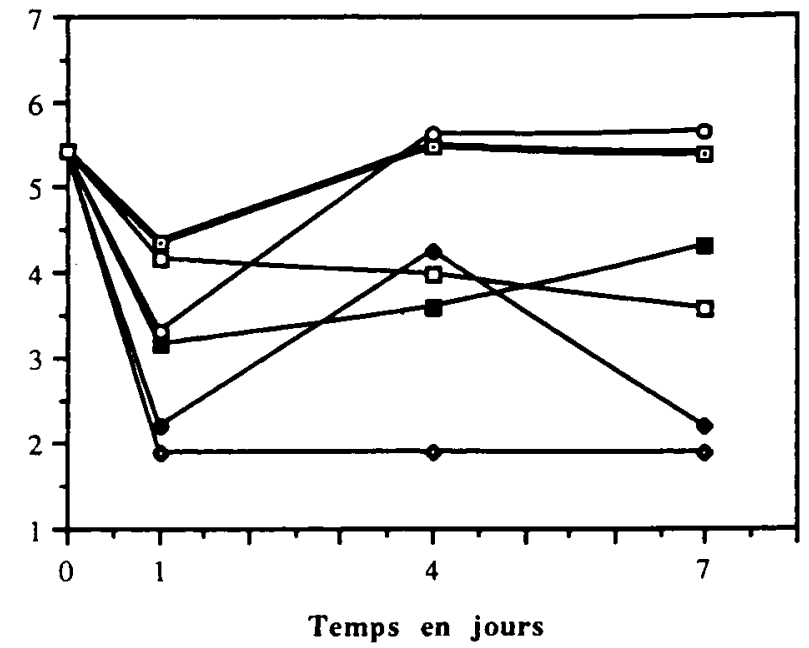

Fig 2. Évolution dans le temps de la population de Ps pv syringae sur plants en acclimatation conduits en microserres en conditions subaseptiques (essai 2) en fonction de différents traitements. - - - témoin eau; - - oxytétra; - - O c cuivre; - 0 - kasugamycine; $-\square$ - fluméquine; $\square \square$ - mancozèbe.

dès le 1 er $\mathrm{j}$; cette action ne persiste cependant pas puisqu'on observe à nouveau une augmentation à $4 \mathrm{j}$. La fluméquine et le mancozèbe entraînent une réduction des populations de 1 à 2 puissances qui se maintient d'ailleurs pendant toute la durée de l'essai. En revanche, la bouillie bordelaise n'a qu'un effet limité sur $P s$ pv syringae. Ce produit se classe, en application du test de Kramer, dans le groupe du témoin eau; la kasugamycine est seule dans un autre groupe significativement différent.

\section{Essai conduit durant l'acclimatation, en conditions normales (essai 3; fig 3)}

La kasugamycine et la fluméquine provoquent une baisse rapide (dès le $1^{\mathrm{er}} \mathrm{j}$ ) et durable des populations qui se maintiennent en dessous de $10^{2}$ bact/feuille. La bouillie bordelaise entraîne également une réduction de l'inoculum dès le $1^{\mathrm{er}}$ j mais le niveau remonte par la suite malgré des variations. L'oxytétracycline montre une efficacité limitée mais constante (en moyenne la population bactérienne est dix fois inférieure à celle du témoin). L'activité du mancozèbe, réelle au cours des 4 premiers jours disparaît totalement par la suite puisqu'au $14 \mathrm{e} j$ on retrouve sur les plants traités un niveau de population voisin de celui des plants témoins. 
Log du nombre de $P$. s. s. par feuille

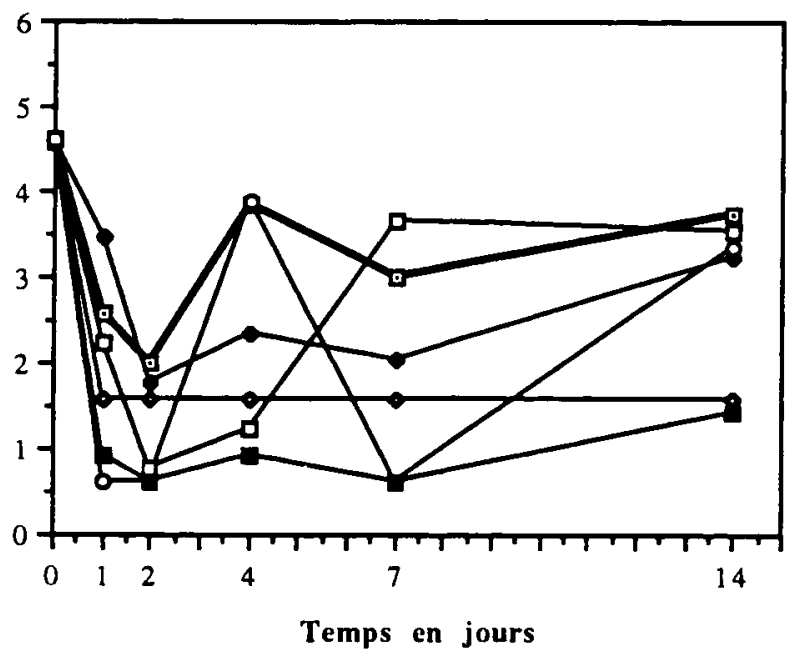

Fig 3. Évolution dans le temps de la population de PS pv syringae sur plants en acclimatation conduits en miniserres en conditions normales (essai 3) en fonction de différents traitements. -๑- témoin eau; - - oxytétra; - - - cuivre; - - - kasugamycine; $-\mathbf{Q}$ - fluméquine; $\square \square-$ mancozèbe.

\section{Essai conduit sur plants en pots (essai 4; fig 4)}

La kasugamycine, et l'oxytétracycline à un niveau moindre, entraînent dès le 1 er $\mathrm{j}$ une réduction des populations, alors que la population des plants témoins est multipliée par 100 environ. La bouillie bordelaise et le mancozèbe sont incapables de contenir la multiplication bactérienne, tout au plus parviennent-ils à la limiter. L'effet de l'application de fluméquine est variable dans le temps : à 3 j les populations concernées sont au niveau de celles des plants témoins mais une réduction significative intervient par la suite. Le test statistique montre que la kasugamycine est le seul produit significativement différent du témoin eau.

\section{DISCUSSION}

L'application de la même statistique d'ordre aux résultats de chacun des 4 essais (tableau I) permet de répartir les produits en 3 groupes. Le premier contient systématiquement le témoin eau. Le troisième, qui correspond aux produits de bonne efficacité, significativement séparé du précédent, on trouve toujours la kasugamycine. Le groupe 2 est intermédiaire et contient des molécules d'efficacité moyenne.
Log du nombre de P. s. s. par feuille

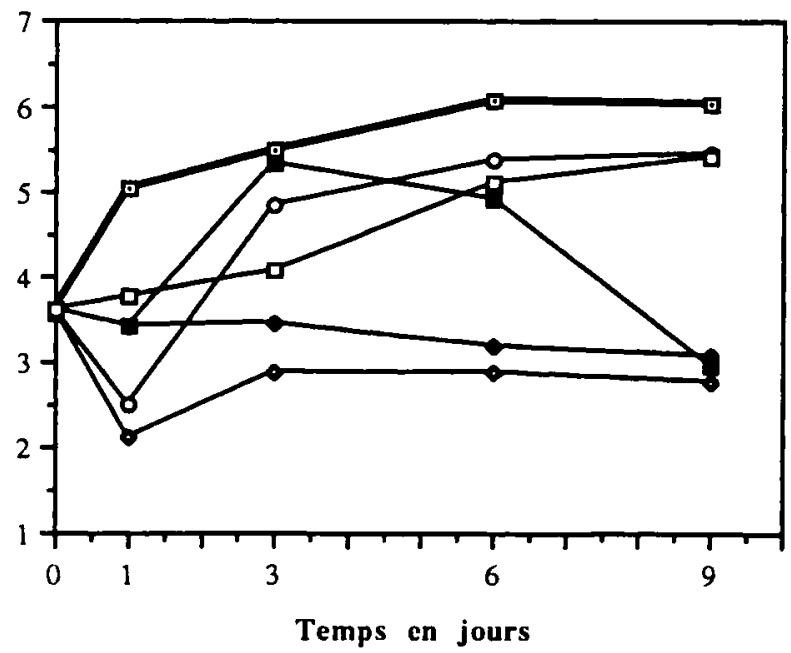

Fig 4. Évolution dans le temps de la population de $P S$ pv $s y-$ ringae sur plants en pots (essai 4) en fonction de différents traitements. -0 - témoin eau; - oxytétra; - - - cuivre; $\rightarrow-$ kasugamycine; - - fluméquine; $-\square-$ - mancozèbe.

Les résultats obtenus sur vitroplants ou sur plants en acclimatation dans les microserres (conditions subaseptiques) et dans les miniserres (conditions normales), ne différent pas significativement de ceux obtenus sur plants de vigne en pots. Une bonne efficacité de la kasugamycine et une efficacité légèrement inférieure de la fluméquine ont été rapportées antérieurement par Luisetti et al (1990) sur vigne et par Brisset et al (1991) sur arbres fruitiers. Une activité moyenne mais variable selon les essais du sulfate de cuivre, a été constatée par plusieurs auteurs et en particulier par Prunier et al (1974) vis-à-vis de $P s$ pv persicae sur pêcher, par Cottin (1989) vis-à-vis de $P s$ pv syringae sur laurier palme et par Luisetti et al (1990) vis-à-vis de $P S$ pv syringae sur vigne. L'amélioration des formulations de sels de cuivre souhaitée par Coléno et al (1982) en vue d'optimiser leur efficacité n'a pas été prise en compte par les industriels; elle devrait concerner à la fois l'efficacité antibactérienne sur plante et la phytotoxicité, et demeure toujours d'actualité. Le vitroplant, très sensible au cuivre, pourrait être utilisé dans le criblage de formulations cupriques modifiées pour une phytotoxicité réduite. L'oxytétracycline qui avait montré une forte activité antibactérienne vis-à-vis de $P s$ pv persicae sur pêcher (Prunier et al, 1974), n'est pas apparue aussi efficace vis-à-vis de $P S$ pv syringae sur vigne dans le cadre de ces essais. Cette différence pourrait être simplement 
due à une baisse d'activité du produit conservé au laboratoire depuis plusieurs années et qu'il n'est plus possible de se procurer même aux États-Unis. Le mancozèbe, produit qui n'est pas connu pour être un bactéricide, a été cependant signalé une seule fois efficace contre $P s$ pv tomato sur des tomates conduites en serre (Conflin et Mc Carter, 1983), il n'a pas montré efficacité notable dans le cadre de nos essais.

\section{CONCLUSION}

Le vitroplant de vigne peut être utilisé pour estimer la capacité de molécules chimiques à réduire le niveau des populations de $P s$ pv syringae présentes à sa surface. Le plant en acclimatation dans une microserre et conduit en conditions subaseptiques serait un outil à développer, entre le test in vitro permettant de déterminer la concentration minimale inhibitrice (CMI) et l'essai de plein champ. Ce matériel pourrait remplacer l'essai parcellaire tel que le pratiquaient Gaignard et al (1973). Le coût étant réduit, les firmes phytosanitaires pourraient alors envisager de tester un grand nombre de molécules nouvelles et de nouvelles formulations de produits moyennement efficaces ou présentant des difficultés d'emploi telles que la phytotoxicité. Les effets de l'application de bactéricides (sels de cuivre ou antibiotiques) sur l'émergence de populations résistantes pourraient être analysées sur ce matériel végétal.

De plus, la feuille de vitroplant pourrait être un bon support pour mener en parallèle des observations en microscopie électronique à balayage ou pour effectuer des microtitrages de produits chimiques afin d'expliquer la bonne efficacité in vitro, non confirmée in situ de certains produits, comme la fluméquine. Cette méthodologie de criblage de molécules chimiques validée sur le modèle vigne/ $P s$ pv syringae est adaptable à tout couple hôte/bactérie dans la mesure où, d'une part, la multiplication de l'hôte in vitro et l'acclimatation du vitroplant sont maîtrisées et, d'autre part, la bactérie pathogène ou glaciogène présente une phase épiphyte dans son cycle biologique; c'est notamment le cas d'un bon nombre de plantes ligneuses ou herbacées et de la plupart des germes des espèces $P$ syringae et Xanthomonas campestris.

\section{REMERCIEMENTS}

Les auteurs remercient l'INRA-Agri-obtentions, Dijon; le laboratoire Ridé, INRA Angers et le GCEV Mumm recherche et vignobles, Epernay qui ont fourni le matériel végétal ainsi que $M$ Durand, étudiante à l'IUT d'Angers qui, au cours d'un stage, a participé à la réalisation des essais et $M$ Bozzini pour la mise en forme de cet article.

\section{RÉFÉRENCES}

Andrews JH (1985) Strategies for selecting antagonistic microorganisms from the phylloplane. In: Biological control of the phylloplane (CE Windels, SE Lindow, eds) Am Phytopathol Soc, St Paul, 31-44

Arny DC, Lindow SE, Upper GD (1976) Frost sensitivity of Zea mays increased by application of Pseudomonas syringae. Nature 262, 282-284

Brisset MN (1990) Possibilités offertes par les techniques de culture in vitro dans la recherche de moyens de lutte contre le feu bactérien. In: Le feu bactérien des Pomoidea (Erwinia amylovora Burill, Winslow et al). CECA-CEE-CEA, Bruxelles, Luxembourg, 81-85

Brisset MN, Luisetti J, Gaignard JL (1991) Firestop: a chemical against bacterial diseases of fruit trees recently available in Europe. Agronomie 11, 93-99

Chabbert YA (1973) Données actuelles sur la résistance des bactéries aux antibiotiques. Actual Pharmacol, $26^{\mathrm{e}}$ sér, Paris

Conflin KC, Mc Carter SM (1983) Effectiveness of selected chemicals in inhibition Pseudomonas syringae pv tomato in vitro and in controlling bacterial speck. Plant Dis 67, 693-644

Coléno A, Gardan L, Luisetti J, Paulin JP (1982) Les antibiotiques et la lutte contre les maladies bactériennes des plantes. In : Les maladies des plantes, $2^{\mathrm{e}}$ journ fr études inf, Paris, octobre 1979, Acta, 355-362

Cottin S (1989) La criblure bactérienne du laurier palme due à Pseudomonas syringae pv syringae. Thèse d'Univ, Univ Angers, 118p

Crosse JE (1959) Bacterial canker of stone-fruits. 4. Investigation of a method for measuring the inoculum potential of cherry trees. Ann Appl Biol 47, 306-317

Dowler WM, Petersen DH (1967) Transmission of Pseudomonas syringae in peach trees by bud propagation. Plant Dis Rep 51, 666-668

English H, Davis JR (1960) The source of inoculum for bacterial canker and blast of stone fruit trees. Phytopathology 50, 634 
Ercolani GL (1969) Sopravvivenza epitifica di populazioni di Pseudomonas morsprunorum Wormald da Ciliego e di $P$ syringae Van hall da Pero sulla pianta ospite di provenirza e sull'altra. Phytopathol Mediterr 8, 197-206

Gaignard JL, Gardan L, Luisetti J, Prunier JP (1973) Schéma d'étude de l'activité antibactérienne de substances vis-à-vis de Pseudomonas morsprunorum f sp persicae. Ann Phytopathol 5, 111

Gardan L, Prunier JP, Luisetti J, Bezelgues JJ (1973) Responsabilité de divers Pseudomonas dans le dépérissement bactérien de l'abricotier en France. Rev Zool Agric Pathol Vég 4, 112-120

Goto M (1991) Plasmids associated with the resistance to copper and streptomycin in Pseudomonas syringae pv actinidiae. Summaries of papers. 4th int working group Pseudomonas syringae pathovars, Florence June 1991, 39

Gross DC, Cody YS, Proebsting EL, Radamaker G, Spotts RA (1983) Distribution, population dynamics and characteristics of ice nucleation active bacteria in deciduous fruit tree orchards. App/ Environ Microbio/ 46, 1370-1379

Hattingh MJ, Roos IMM, Mansvelt EL (1989) Infection and systematic invasion of deciduous fruit trees by Pseudomonas syringae in South Africa. Plant Dis 73, 784-789

Hirano SS, Hirano EA, Kelman A, Upper CD (1978) Ice nucleation activity of fluorescent plant pathogenic pseudomonads. Proc 4th Int Conf Plant Bact, 717-725, Angers 1978

Kahan G, Cooper D, Papavasiliou A, Kramer A (1973) Expanded tables for determining significance of differences of ranked data. Food Technol 27, 63-69

King EO, Ward MK, Raney DE (1954) Two simple media for the demonstration of pyocyanin and fluorescin. J Lab Clin Med 44, 301-307

Leben C (1985) Introductory remarks: biological control strategies in the phylloplane. In: Biological control of the phylloplane (CE Windels, SE Lindow ed). Am Phytopathol Soc, St Paul 1-5

Lindow SE (1983) Methods of preventing frost injury caused by epiphytic ice-nucleation active bacteria. Plant Dis 67, 327-333

Lindow SE (1988) Lack of correlation of in vitro antibiosis with antagonism of ice nucleation active bacteria on leaf surfaces by non-ice nucleation active bacteria. Phytopathology 78, 444-450

Lindow SE (1991) Copper tolerance of Pseudomonas syringae strains from fruit trees in California. Summaries of papers, 4th Int Work Group on Pseudomonas syringae pathovars, Florence, June 1991, 45

Lindow SE, Connel JH (1984) Reduction of frost injury to almond by control fo ice nucleation active bacteria. J Am Soc Hortic Sci 109, 48-53

Lindow SE, Arny DC, Upper CD (1978a) Bacterial ice nucleation: a factor in frost injury to plants. Plant Physiol 70, 1084-1089
Lindow SE, Arny DC, Barchet WR, Upper CD (1978b) The role of bacterial ice nuclei in frost injury to sensitive plants. In: Plant cold hardiness and freezing stress (Li pH, Sakai A, eds) Acad Press Inc, New York, 249-261

Luissetti J, Paulin JP (1972) Étude sur les bactérioses des arbres fruitiers. 3. Recherche de Pseudomonas syringae à la surface des organes aériens du Poirier et étude de ses variations quantitatives. Ann Phytopathol 4, 215-227

Luisetti J, Gaignard JL (1986) Les bactéries glaçiogènes et le gel de printemps. Viti $97,13-14$

Luisetti J, Gaignard JL (1987a) Bacterial populations in buds and probability of frost injury on grapevine. Proc 6th Int Conf Plant Pathogenic Bacteria, Maryland, June 1985. Martinus Nijhoff Publishers Dordrecht, 986-993

Luisetti J, Gaignard JL (1987b) Deux maladies bactériennes du kiwi en France. Phytoma 391, 42-45

Luisetti J, Gaignard JL, Pacqueteau B, Lafuste JP (1984) Le dépérissement du pêcher. Phytoma 358, 29-32

Luisetti J, Gaignard JL, Ridé M (1990) Ability of kasumin to control some phytopathogenic bacteria. Proc 7th Conf Plant Pathogenic Bacteria, Budapest, June 1989 (Z Klement, ed) 213-218

Luisetti J, Gaignard JL, Devaux M (1991) Pseudomonas syringae pv syringae as one of the factors affecting the ice nucleation of grapevine buds in controlled conditions. J Phytopathol 133, 4, 334-344

Maki LR, Willoughby KJ (1978) Bacteria as biogenic sources of freezing nuclei. $J$ Appl Meteorol 17, 1049-1053

Maki LR, Gaylan EL, Chang-Chien MM, Cadwell DR (1974) Ice nucleation induced by Pseudomonas syringae. App/ Microbiol 28, 456-459

Malvick DK, Moore LW (1988) Population dynamics and diversity of Pseudomonas syringae on maple and pear trees and associated grasses. Phytopathology $78,1366-1370$

Manceau C (1984) Utilisation des antibiotiques en agriculture: étude des risques potentiels de sélection et de dissémination des gènes d'antibiorésistance et caractérisation du support génétique de ces caractéres. Thèse de $3^{e}$ cycle, Univ Clermont-Ferrand, $113 p$

Mansvelt EL, Hattingh Mj (1988) Resident populations of Pseudomonas syringae pv syringae on leaves, blossoms and fruits of apple and pear trees. J Phytopathol 121, 135-142

Murashige T, Skoog F (1962) A revised medium for rapid growth and biossays with tobacco tissue. Physiol Plant 15, 473-497

Paulin JP, Luisetti J (1978) Ice nucleation activity among phytopathogenic bacteria. Proc 4th Conf Plant Path Bact 725-731

Prunier JP, Luisetti J, Gardan L (1974) Étude sur les bactérioses des arbres fruitiers, essais de lutte chi- 
mique contre le dépérissement bactérien du pêcher en France. Phytiatr Phytopharm 23, 71-88

Sobiczewski P, Chiou CS, Jones AL (1991) Streptomycin-resistant epiphytic bacteria with homologous DNA for streptomycin resistance in Michigan apple orchards. Plant Dis 75, 1110-1113

Sundin GW, Jones AL, Fulbright DW (1989) Copper resistance in Pseudomonas syringae from cherry orchards and its associated transfer in vitro and in planta with plasmid. Phytopathology 79, 861-865
Vali G, Christensen M, Fres RW, Gaylan EL, Maki LR, Schnell RC (1976) Biogenic ice nuclei. Part II : bacterial sources. J Atmos Sci 33, 1565-1570

Yankofsky SA, Levin Z, Moshe A (1981) Association with Citrus of ice-nucleating bacteria and their possible role as causative agents of frost damage. Curr Microbiol 4, 213-217

Young JM (1978) Survival of bacteria on Prunus leaves. Proc 4th Int Conf Plant Path Bact, Angers, $1978,779-786$ 